This document is the accepted manuscript version of the following article:

Hu, H., Liu, M., Kong, Y., Mysuru, N., Sun, C., Gálvez-Vázquez, M. de J., ...

Broekmann, P. (2020). Activation matters: hysteresis effects during electrochemical looping of colloidal Ag nanowire catalysts. ACS Catalysis, 10(15), 8503-8514. https://doi.org/10.1021/acscata1.0c02026

\title{
Activation matters: hysteresis effects during electrochemical looping of colloidal Ag nanowire (Ag-NW) catalysts
}

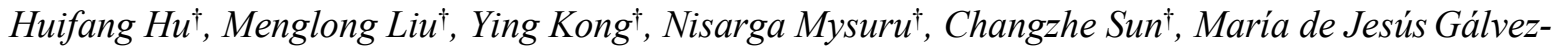

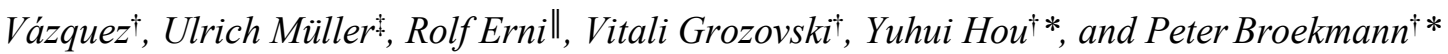 \\ †Department of Chemistry and Biochemistry, University of Bern, Freiestrasse 3, Bern 3012 \\ Switzerland \\ tSurface Science and Coating Technology, Empa - Swiss Federal Laboratories for Materials Science \\ and Technology, 8600 Dübendorf, Switzerland \\ $\|$ Electron Microscopy Center, Empa, Swiss Federal Laboratories for Materials Science and \\ Technology, Überlandstrasse 129, 8600 Dübendorf, Switzerland
}

\begin{abstract}
Colloidal electrocatalysts are commonly synthesized using organic capping agents (surfactants), which control the size distribution and shape of the resulting nano-objects and prevent them from agglomerating during and after synthesis. However, the presence of a surfactant shell on the catalyst is detrimental, as the resulting performance of the electrocatalyst depends crucially on the ability of reactants to access active surface sites. Techniques for post-synthesis deprotection are therefore mandatory for removing the capping agents from the otherwise blocked reactions sites without compromising the structural integrity of the nano-catalysts. Herein, we present silver nanowires (AgNWs) — produced via PVP-assisted polyol synthesis — as effective catalysts for the electrochemical $\mathrm{CO}_{2}$ reduction reaction $\left(e c-\mathrm{CO}_{2} \mathrm{RR}\right)$, which reach Faradaic efficiencies close to $100 \%$ for $\mathrm{CO}$ formation after deprotection by a so-called 'electrochemical looping' (ec-1) pretreatment. Electrochemical looping refers to a sequence of potentiostatic $\mathrm{CO}$ electrolysis experiments that exhibit well-defined starting $\left(\mathrm{E}_{\text {start }}\right)$, vertex $\left(\mathrm{E}_{\mathrm{vertex}}\right)$, and end potentials $\left(\mathrm{E}_{\text {end }}\right)$. The resulting product distribution undergoes a profound hysteresis in the forward and corresponding backward run of the electrochemical looping experiment, thus pointing to an effective deprotection of the catalyst as evidenced by post-electrolysis XPS inspection. These results can be considered as prime example demonstrating the importance of the catalyst's 'history' for the resulting ec- $\mathrm{CO}_{2} \mathrm{RR}$ performance. These transient (non-steady-state) effects are crucial in particular for the initial stage of the $\mathrm{CO}_{2}$ electrolysis reaction and for catalyst screening approaches carried out on the time scale of hours.
\end{abstract}


Key words: $\mathrm{CO}_{2}$ reduction reaction; silver nanowires; surfactant removal; catalyst deprotection; electrochemical looping

\section{Introduction}

The conversion of environmentally harmful carbon dioxide $\left(\mathrm{CO}_{2}\right)$ into value-added products is one of the major intersectoral challenges that we currently face. ${ }^{1}$ In this context, electrochemical approaches of $\mathrm{CO}_{2}$ valorisation deserve particular attention as they can utilize the 'green' electric power generated by renewables such as wind, solar, or wind energy — as energy input for the highly endergonic process of $\mathrm{CO}_{2}$ electrolysis, thereby rendering the overall process more sustainable. ${ }^{2-4}$ One of the main target products of the electrochemical $\mathrm{CO}_{2}$ reduction reaction (hereafter referred to as ec$\mathrm{CO}_{2} \mathrm{RR}$ ) is carbon monoxide ( $\mathrm{CO}$ ), which is currently produced on an industrial scale via the

'Boudouard' reaction and reaches a yearly production volume of approximately $210000 \mathrm{Mt}^{5} \mathrm{CO}$ is considered to be a valuable intermediate (current market price: $\approx 0.65 \$ \mathrm{~kg}^{-1}$ ) and has the potential to be used as a reactant on a large scale (e.g. in the Fischer-Tropsch synthesis of aliphatic hydrocarbons [synthetic fuels] or alcohols). ${ }^{6}$ Cost estimates suggest that the electrochemical co-electrolysis of water $/ \mathrm{CO}_{2}$ might indeed become competitive with more well established routes of $\mathrm{CO}$ production. ${ }^{5}$ The electrochemical production of $\mathrm{CO}$ via the co-electrolysis of water/ $\mathrm{CO}_{2}$ can be considered to be a versatile 'synthesis module', which also can be coupled to other process units for the production of valuable end products. A promising alternative to interlinking this process to the heterogeneous gasphase Fischer-Tropsch reaction has recently been proposed by the Siemens/Evonik consortium ${ }^{1,7}$, and couples the $e c-\mathrm{CO}_{2} \mathrm{RR}$ (CO production; Siemens) to a biotechnological fermentation process (Evonik), thereby yielding fine chemicals such as butanol and hexanol as key intermediates for the production of speciality plastics. ${ }^{7}$ The first test plant is expected to become operative in $2021^{1}$, and thus demonstrates the enormous efforts that are currently underway to bring the $e c-\mathrm{CO}_{2} \mathrm{RR}$ process from the lab to the market. ${ }^{1}$

Catalysts are essential for $e c-\mathrm{CO}_{2} \mathrm{RR}$, as they direct the electrolytic reaction towards the desired target product (e.g. CO). The pioneering work by Hori et al. ${ }^{8-10}$ identified silver $(\mathrm{Ag})$ as one of best (electro)catalysts, in addition to $\mathrm{Au}$ and $\mathrm{Zn}$, which yielded $\mathrm{CO}$ with faradaic efficiencies (FEs) that reached $81.5 \%(E=-1.14 \mathrm{~V}$ vs. $\mathrm{NHE}) .{ }^{8}$ In these early studies, catalyst screening was mainly based on 
the use of polycrystalline electrode materials as active catalysts (e.g. metal foils). However, substantial progress has been made during the last two decades in the development of tailored nano-materials with an improved surface-to-volume ratio and well-defined shapes, the latter being important for the rational design of active surface sites. ${ }^{11-12}$ In future, these nanomaterials have the potential to be used in gas diffusion electrodes (GDEs) as a key component of advanced gas-flow electrolyser systems. ${ }^{7}$, 13${ }^{16}$ From a technical point of view, it is mandatory to use a gas-flow approach to reach the current densities that are targeted by industry (100 to $\left.1000 \mathrm{~mA} \mathrm{~cm}^{-2}\right)$ to cover the capital and operating costs of these systems. ${ }^{13,17-18}$ Typically, these nanomaterials are produced via colloidal synthesis, which also allows the process to be easily scaled up and which is considered to be a key perquisite for any industrial application. A wide range of particle morphologies can be obtained using this colloidal approach, ranging from spheres ${ }^{19}$, cubic shapes ${ }^{20}$, and triangular platelets (confined 2-D systems) ${ }^{21}$ to 1-D nano-objects such as rods ${ }^{19}$ and wires ${ }^{22-26}$. To rationally design these nano-objects, a multiparameter space needs to be considered, which includes but is not limited to: (i) the reaction temperature, (ii) the convective transport of reactants (e.g. stirring speed), (iii) the ratio of reactants (e.g. metal ion precursor, reducing agent, etc.), (iv) reaction times, (v) and the injection speed of chemicals. ${ }^{27-29}$ However, the most important aspect to consider is the action of the so-called surfactants and capping agents. ${ }^{19,25,30}$ Their presence in the reaction medium crucially affects the nucleation and growth kinetics of the nano-objects and could even cause crystal growth to be anisotropic, which is required for the synthesis of metallic nanowires (NWs). ${ }^{31}$ The physical origin of this anisotropic growth behaviour is the preferential surfactant adsorption on certain surface facets (e.g., [100] textured), which reduces their growth rate relative to surfaces with different surface orientations (e.g., [111]). ${ }^{30,32-35}$ In this sense, the role of the surfactants is a result of the steric blocking of surface sites that are active for the (e-less) metal deposition by selectively limiting the access of precursor metal ions in the liquid reaction medium to the emerging surface of the nano-crystals (NCs). ${ }^{36}$ Note that not only the monomeric ${ }^{11,37}$ or polymeric (e.g. $\mathrm{PVP}^{38}$ ) organic surfactants need to be considered, but also anionic species (e.g. halides) that are added to the reaction media along with the metal precursors. These counter anions usually play a crucial role in the initial nucleation process of the nano-objects 
(concept of self-seeded growth ${ }^{25,35}$ ) and further tend to chemisorb on the emerging facets in an advanced stage of $\mathrm{NC}$ growth ${ }^{31}$.

While this facet-specific blocking by adsorbed surfactants is a mechanistic prerequisite for any anisotropic growth mode, it is highly detrimental to the desired (electro)catalytic performance of the nano-catalysts. As capping agents sterically block the access of reactants to the active catalyst sites during the electrocatalysed reaction of interest, ${ }^{37-40}$ various 'soft' post-synthesis methods have been proposed to deprotect the 'capped' nano-objects without compromising their structural integrity (e.g. loss of the shape, changes in size distribution, NP agglomeration, etc.). These deprotection techniques range from purely physical (e.g. thermal annealing ${ }^{41-42}$ or exposure to light of particular wavelength and intensity ${ }^{43-44}$ ) to chemical treatments under non-reactive (e.g. 'chemical' washing ${ }^{38}$ ) or reactive conditions (e.g. plasma treatment, the use of oxidizing or reducing agents, etc.) ${ }^{37-38,44-51}$. Note that, under extremely drastic experimental conditions (e.g. thermal treatment at elevated temperatures), this type of catalyst pretreatment could lead to the loss of surface texture or to the agglomeration of nanoparticles. $^{52}$

Also electrochemical treatments (anodic or cathodic polarization) have successfully been applied to deprotect colloidal catalysts. ${ }^{53-56}$ For example, Oezaslan et al. ${ }^{54}$ reported on the efficient removal of a PVP capping shell from Pt nano-cubes by applying an oxidative stressing protocol (electrochemical cycling up to $+0.8 \mathrm{~V}$ vs. RHE in $0.1 \mathrm{M} \mathrm{HClO}_{4}$ ), whereas the electrochemical deprotection failed under alkaline conditions. Also the chemical nature of the capping agent (PVP versus oleylamine) has been shown to play a crucial role in the structural integrity of the nano-catalysts after electrochemical deprotection. ${ }^{54}$

So far, most studies on catalyst activation have considered only one single electrocatalytic reaction, (e.g. the oxygen reduction reaction $[\mathrm{ORR}]^{53-54}$, the oxygen evolution reaction $[\mathrm{OER}]$, or the hydrogen evolution reaction $[\mathrm{HER}]^{37}$ etc.). For these single reactions, there are straightforward electrochemical descriptors and measuring approaches available to monitor the effectiveness of the applied deprotection technique (e.g. via the electrochemically active surface area [ECSA]), which is probed either by faradaic or non-faradaic processes. Their increase is directly proportional to the increase in the ECSA and is related to an overall improvement in the reaction rate. ${ }^{37,40,49,53-54}$ 
However, the situation is more complex when considering the $e c-\mathrm{CO}_{2} \mathrm{RR}$ owing to the fact that the $\mathrm{CO}_{2}$ electroreduction is necessarily superimposed on the parasitic HER when carried out in an aqueous reaction environment, which leads to a less-than-unity Faradaic efficiency of the $e c-\mathrm{CO}_{2} \mathrm{RR}$. Thus, the presence of the capping agents and the applied deprotection treatment do not only affect the overall reaction rate (current density normalized to the geometric surface area), but also the resulting product distribution.

Herein, we present a comprehensive study on an approach to electrochemical catalyst activation (surfactant removal) that utilizes the $e c-\mathrm{CO}_{2} \mathrm{RR}$ itself to achieve the desired catalyst deprotection. As catalyst of choice, we applied silver nanowires (Ag-NWs) that were synthesized by a self-seeding polyol process using high molecular weight PVP as the capping agent. ${ }^{24,27,32,57-58}$ The co-electrolysis of water/ $\mathrm{CO}_{2}$ that is performed over $\mathrm{Ag}$ catalysts yields only $\mathrm{H}_{2}$ and $\mathrm{CO}$ as the reaction products. ${ }^{8-10,59}$ In the present study, we sought to demonstrate that the formed $\mathrm{CO}$ acts as an excellent surfactant removal agent that is capable of deprotecting the Ag-NWs, thereby further self-accelerating the ec$\mathrm{CO}_{2} \mathrm{RR}$ at the expense of the parasitic HER and leading to CO efficiencies of nearly $100 \%$. This PVP removal by 'cathodic' electrode polarization complements the 'oxidative' approach that was proposed by Oezaslan et al. ${ }^{54}$

\section{Experimental}

Catalyst synthesis - Ag nanowires (Ag-NWs) were synthesized in a three-necked flask according to a modified protocol introduced by Jiu et al. and others. ${ }^{24,27,32,57-58}$ For this purpose, $0.2 \mathrm{~g}$ of PVP $\left(\mathrm{M}_{\mathrm{w}}=\right.$ $1300000 \mathrm{~g} \mathrm{~mol}^{-1}$, Sigma-Aldrich; see Figure 1) was dissolved at room temperature under magnetic agitation in $25 \mathrm{~mL}$ of ethylene glycol (EG, Sigma-Aldrich, 99.8\%). Subsequently, $0.25 \mathrm{~g}$ of silver nitrate $\left(\mathrm{AgNO}_{3}\right.$, Sigma-Aldrich, ACS reagent, $\left.\geq 99.8 \%\right)$ was added to the PVP containing EG, followed by the addition of a solution of $1.95 \mathrm{mg} \mathrm{FeCl}_{3}$ (Sigma-Aldrich, 97\%) pre-dissolved in $2 \mathrm{~mL}$

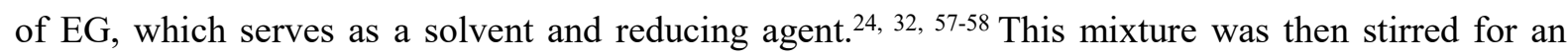
additional 2 minutes before the three-necked flask containing the transparent EG solution was transferred to a pre-heated oil bath. This solution was kept at $130^{\circ} \mathrm{C}$ for a total of 5 hours. During the first hour, the solution was continuously stirred, while no magnetic agitation was applied during the 
last 4 hours of the thermal treatment. The resulting Ag-NW precipitate was separated from the EG solvent by centrifugation at $4000 \mathrm{rpm}$ for $10 \mathrm{~min}$, followed by three repetitive washing/centrifugation treatments using a mixture of Milli-Q water and acetone $\left(\mathrm{V}_{\text {water }}: \mathrm{V}_{\text {aceton }}=2: 1\right)$, ultimately yielding 24 mg of the Ag-NW catalyst (denoted 'as-synthesized'). The Ag-NW powder was finally redispersed in $8 \mathrm{~mL}$ of isopropanol (BASF SE, assay $\geq 99.0 \%$ ).

Electrode preparation - After 30 min of sonication, $50 \mu \mathrm{L}$ of the $\mathrm{Ag}-\mathrm{NW}$ suspension was drop-casted onto a glassy carbon support electrode ( $\mathrm{A}=0.8 \mathrm{~cm}^{-2}$, Alfa Aesar, $2 \mathrm{~mm}$ thickness).

For the sake of comparison, Ag-NW catalysts were also dispersed onto a technical carbon support. For this purpose, $12 \mathrm{mg}$ of the as-prepared Ag-NWs was suspended in $15 \mathrm{~mL}$ of isopropanol, followed by $1 \mathrm{~h}$ of sonication. $12 \mathrm{mg}$ of technical carbon powder (Vulcan XC 72R, Cabot, USA) was dispersed in $15 \mathrm{~mL}$ of isopropanol, and was also followed by $1 \mathrm{~h}$ of sonication. Both suspensions were

subsequently mixed and homogenized by sonicating for $30 \mathrm{~min}$. The resulting suspension was dried under vacuum conditions and yielded a carbon-supported (C-supported) Ag-NW catalyst powder. This powder was redispersed in $4 \mathrm{~mL}$ of isopropanol containing $400 \mu \mathrm{L}$ of Nafion solution (Aldrich, $5 \mathrm{wt} \%$ dissolved in a mixture of lower aliphatic alcohols and water) and subjected to 30 min of sonication. Subsequently, $50 \mu \mathrm{L}$ of the resulting ink was drop-casted onto the glassy carbon support electrode (see aforementioned protocol).

Electrode characterization - The morphology of the Ag-NW films (non-supported, C-supported) that were deposited on the glassy carbon support electrodes were characterized by means of Scanning Electron Microscopy (Zeiss Gemini SEM450). Complementary white-light interferometry (ContourGT profilometer, Bruker) was applied to determine the thickness and roughness of the AgNW films. For the transmission electron microscopy (TEM) imaging and selective area electron diffraction, an FEI Titan Themis instrument was used with an accelerating voltage of $300 \mathrm{kV}$. An x-ray photoelectron (XPS) inspection was performed on a Physical Electronics (PHI) Quantum 2000 Scanning ESCA Microprobe System using monochromated Al K $\alpha$ radiation $(\mathrm{h} v=1486.7 \mathrm{eV})$. A hemispherical capacitor electron-energy analyser, equipped with a channel plate and a positionsensitive detector, was operated under an electron take-off angle of $45^{\circ}$. For the acquisition of the high-resolution $\mathrm{Ag} 3 d, \mathrm{Cl} 2 p$ and $\mathrm{N} 1 s$ photo-emission data, the analyser was operated with a constant 
pass energy mode at $23.5 \mathrm{eV}$ and an energy step width of $0.20 \mathrm{eV}$. The x-ray beam diameter was around $150 \mu \mathrm{m}$. The binding energy was calibrated using the $\mathrm{Cu} 2 p_{3 / 2}, \mathrm{Ag} 3 d_{5 / 2}$, and $\mathrm{Au} 4 f_{7 / 2}$ emissions at $932.62 \mathrm{eV}, 368.21 \mathrm{eV}$, and $83.96 \mathrm{eV}$, respectively, to within $\pm 0.1 \mathrm{eV}$ [see ISO 15472; 2010-05]. Built-in electron and argon ion neutralizers were applied in order to compensate for eventual surface charging effects. The base pressure of the XPS system was below $5 \times 10^{-7} \mathrm{~Pa}$. The XPS spectra were analysed using the MultiPak 8.2B software package and were subjected to a Shirley background subtraction. The atomic concentrations were determined based on the corrected relative sensitivity factors that were provided by the manufacturer and normalized to 100 at $\%$. The uncertainty was estimated to be ca. $10 \%$.

Electrochemical experiments - For all electrochemical experiments, a potentiostat/galvanostat (Metrohm Autolab 302N, The Netherlands) was used to control the potential, current density, and transferred charge. The electrolysis experiments were carried out using a custom-built, air-tight, glasscell (H-type) as previously described (see Figure S1). ${ }^{60-62}$ For the iR compensation, cell resistance was determined by means of impedance spectroscopy (FRA module, Autolab Nova). Hence, all potentials provided herein are iR-compensated to $\sim 85 \%$ of the measured cell-resistance.

The three-electrode arrangement used here consisted of a leakless $\mathrm{Ag} / \mathrm{AgC}_{3 \mathrm{M}} 1$ electrode (Pine), a bright Pt-foil (15 $\mathrm{mm} \times 5 \mathrm{~mm}$ ), and the Ag-NW catalyst film (non-supported, C-supported) served as the reference, counter, and working electrodes, respectively.

For the sake of comparability, all potentials measured versus $\mathrm{Ag} / \mathrm{AgCl}_{3 \mathrm{M}}$ are referenced herein with respect to the reversible hydrogen electrode (RHE). The applied potentials (vs. $\mathrm{Ag} / \mathrm{AgCl}_{3 \mathrm{M}}$ ) were converted to the RHE scale using the following equation:

$$
\mathrm{E}_{\mathrm{RHE}}(\mathrm{V})=\mathrm{E}_{\mathrm{Ag} / \mathrm{AgCl}(3 \mathrm{M})}(\mathrm{V})+0.210 \mathrm{~V}+(0.059 \mathrm{~V} \times \mathrm{pH})
$$

Note that the anolyte and the catholyte were separated by a Nafion 117 membrane (Figure S1). This cell design also prevents the transfer of trance amounts of Pt ions from the anolyte to the catholyte when using Pt as the material for the counter electrode (see reference measurements presented in Figure S2-S5) as evidenced by ICP-MS measurements (NexION 2000 ICP-MS instrument, Perkin Elmer). Also note that no change of the $e c-\mathrm{CO}_{2} \mathrm{RR}$ product distribution is observed when exchanging the Pt counter electrode by Ir (see Figure S5). 
Electrolysis experiments were carried out in $0.5 \mathrm{M} \mathrm{KHCO}_{3}$ (ACS grade, Sigma-Aldrich) electrolyte solutions that were saturated either with $\mathrm{Ar}$ (blank) or $\mathrm{CO}_{2}$ gas (99.999\%, Carbagas, Switzerland). The $\mathrm{pH}$ of the $\mathrm{CO}_{2}$ - and Ar-saturated $0.5 \mathrm{M} \mathrm{KHCO}_{3}$ was 7.5 and 8.9 , respectively.

Technical details of the $\mathrm{CO}_{2} \mathrm{RR}$ product analysis based on online gas-chromatography have been previously described. ${ }^{60-62}$ A so-called single-catalyst approach was applied in order to demonstrate the pronounced hysteresis effects on the potential-dependent $\mathrm{CO}_{2} \mathrm{RR}$ product distribution. ${ }^{63}$ The same electrode was used for a defined sequence of potentiostatic electrolysis experiments, which differed in both the electrolysis time and the width of the potential window applied to the catalyst. In a further step a multi-catalyst approach was applied ${ }^{63}$, in which a newly prepared (pre-conditioned) catalyst was used for each applied electrolysis potential to demonstrate the performance of the deprotected Ag-NW catalysts.

\section{Results and discussion}

Structural characterization - Figure 2 displays top-down SEM images of the two types of Ag-NW catalysts used in this study. A three-dimensional network of randomly-distributed and loosely-packed Ag-NWs is formed after drop-casting the Ag-NW suspension on the glassy carbon support electrode (Figure 2 panels a-c). Complementary white light interferometry reveals a homogeneous layer of AgNWs on the glassy carbon electrode with a root mean square (RMS) roughness and film thickness of $76 \mathrm{~nm}$ and $885 \mathrm{~nm}$, respectively (Figure S6a-c). On the nm length scale the network of Ag-NWs shows a more inhomogeneous appearance. Note that the surface of the glassy carbon support remains visible in the top-down SEM inspection (Figure 2c). Therefore, the entirety of the Ag-NW film is, when exposed to the aqueous environment, likely to be wetted by the electrolyte down to the glassy carbon electrode. A statistical analysis of the SEM images reveals that the mean thickness of the AgNWs is approximately $162 \mathrm{~nm}$ (inset of Figure 2a), whereas they range in length from ca. one to several microns. According to the literature, ${ }^{22,}{ }^{64-65}$ the Ag-NWs exhibit a five-fold twinned facecentred cubic (fcc) structure with a preferential orientation along the (110) crystallographic direction. The sidewalls of the Ag-NWs consist of five (100) textured facets, whereas the pentagonal apex of the Ag-NW is (111) terminated. These hexagonal facets represent the actual growth front in the Ag-NW 
synthesis in which the monovalent $\mathrm{Ag}^{+}$precursor ions are reduced and added to the developing nanowire. The origin of this highly anisotropic metal growth is the chemisorption of additives/surfactants (e.g. chloride and PVP), which is supposed to be weaker on the (111) facets, thereby rendering them more active for the e-less metal deposition than the (100) facets. ${ }^{58}$ The latter experience a steric blocking by the more strongly chemisorbed surfactants. ${ }^{22,66}$

One drawback of the Ag-NW model catalyst drop-casted on the glassy carbon support is the potential loss of catalyst material during extended electrolysis, in particular when the electrolysis reaction involves massive gas evolution, e.g. by the parasitic HER that is inevitably superimposed on the $\mathrm{CO}_{2} \mathrm{RR}$ in an aqueous environment. ${ }^{43}$ This loss of catalyst material is a result of the weak adhesion of the NW layer to the glassy carbon support electrode and the loose packing of the Ag-NWs inside the catalyst film. One possible approach to circumventing this structural degradation is based on the mechanical stabilization of the NW film. This stabilization can be achieved by the use of a technical carbon support (e.g. Vulcan) in combination with a Nafion binder, thereby substantially improving both the adhesion of the catalyst film to the glassy carbon support and the cohesion inside the film. ${ }^{67}$ Figure 2d-f depicts the corresponding top-down SEM images of the C-supported Ag-NW catalysts that were drop-casted on the glassy carbon electrode, demonstrating that individual Ag-NWs were embedded in the highly porous carbon support. However, one possible drawback of this approach could be an increase in the contribution of the porous carbon material to the resulting product distribution in the form of an increase in the parasitic HER (see discussion of Figure 6 below). An alternative approach to catalyst stabilization, which is based on a so-called photonic curing, has recently been introduced by Hou et al.. ${ }^{43}$ This treatment induces a local melting and subsequent solidifying of the NWs at their points of contact. Photonically-cured free-standing films of metallic nanowires were demonstrated to resist even massive gas-evolution reactions without any indication of structural degradation..$^{43}$

Electrochemical activation of Ag-NW catalysts by electrochemical looping - The working hypothesis, which was to be verified in the following experiments, is that the surfactants (i.e. chloride and PVP) on the Ag surface of the deposited nanowires severely affect the product distribution of ec- $\mathrm{CO}_{2} \mathrm{RR}$, and undergo substantial alterations over the course of the performed co-electrolysis reaction. An 
efficient catalyst activation that is induced by the electrolysis reaction itself can be deduced from the pronounced hysteresis characteristics observed in the potential-dependent product distribution, which is displayed in Figure 3 as Faradaic efficiency versus applied potential ( $F E$ versus $E$ ) plots (Table $\mathrm{S} 1$ ). These dedicated electrolysis experiments are referred to as 'electrochemical looping' (ec-l), in which the applied electrolysis potentials of the individual 40 min-long electrolyses were changed in a stepwise manner from a fixed starting point of $E_{\text {start }}=-0.6 \mathrm{~V}$ vs. RHE to a variable 'lower' vertex potential $\left(\mathrm{E}_{\text {vertex }}\right.$ ) that ranged from $-0.9 \mathrm{~V}$ vs. RHE to $-1.3 \mathrm{~V}$ vs. RHE (Figure 3a-e). The electrolysis loop is closed through the corresponding backward run of electrolysis experiments, and ends at the initial starting potential $\left(\mathrm{E}_{\text {start }}=\mathrm{E}_{\text {end }}\right)$. The main products of the electrolysis in the $\mathrm{CO}_{2}$-saturated $0.5 \mathrm{M}$ $\mathrm{KHCO}_{3}$ aqueous solution are $\mathrm{CO}$ (black circles, Figure 3) and $\mathrm{H}_{2}$ (red squares, Figure 3). The filled and non-filled circles/squares refer to FE values, which correspond to the forward and the corresponding backward runs of the electrochemical looping campaigns. As long as the lower vertex potential remains larger than or equal to $-0.9 \mathrm{~V}$ vs. RHE (Figure 3a), only a marginal deviation is observed in the product distributions of the forward and the corresponding backward electrolysis runs (see also Figure S7). However, a minor trend towards increased $\mathrm{CO}$ efficiencies (decreased $\mathrm{H}_{2}$ efficiencies) can be observed in the backward run. This positive trend of catalyst activation is continued by further shifting the lower vertex potential to more negative applied electrolysis potentials (Figure $3 \mathrm{~b}-\mathrm{e}$ ). When extending the potential window of electrolysis to a vertex potential of $\mathrm{E}_{\mathrm{vertex}}=-$ $1.3 \mathrm{~V}$ vs. RHE, CO efficiencies of $>80 \%$ were achieved in the corresponding backward electrolysis run (Table S1e). In general, the shape of the product distribution in the $F E$ versus $E$ plot in Figure $3 \mathrm{e}$ displays an anti-correlated change in the $\mathrm{FE}$ values for $\mathrm{CO}$ and $\mathrm{H}_{2}$, which exceed the maximum in $\mathrm{CO}$ efficiency (minimum in $\mathrm{H}_{2}$ efficiency) at potentials between -1.0 and $-1.1 \mathrm{~V}$ vs. RHE (forward run). Interestingly, a more extended plateau of approximately $300 \mathrm{mV}$ develops in the corresponding backward run in the potential range of $-1.1 \mathrm{~V}$ to $-0.8 \mathrm{~V}$ vs. RHE, ultimately reaching $\mathrm{CO}$ efficiencies of $>80 \%$.

The $\mathrm{FE}_{\mathrm{CO}}$ and $\mathrm{FE}_{\mathrm{H} 2}$ values were the most substantially impacted by electrochemical looping at medium and low overpotentials ( $>-1.1 \mathrm{~V}$ vs. RHE), whereas only minor differences were observed in the forward and backward runs for applied electrolysis potentials of $<-1.2 \mathrm{~V}$ vs. RHE (Figure 3e). In 
Figure $3 \mathrm{e}$, the differences in potentials between the backward and the respective forward runs were $\Delta \mathrm{FE}_{\mathrm{CO}}=+10.3 \%$ at $-1.0 \mathrm{~V}$ vs. RHE, $\Delta \mathrm{FE}_{\mathrm{CO}}=+32.2 \%$ at $-0.9 \mathrm{~V}$ vs. RHE, $\Delta \mathrm{FE}_{\mathrm{CO}}=+60.0 \%$ at $-0.8 \mathrm{~V}$ vs. RHE, $\Delta \mathrm{FE}_{\mathrm{C}}=+71.4 \%$ at $-0.7 \mathrm{~V}$ vs. RHE, and $\Delta \mathrm{FE}_{\mathrm{CO}} \quad=+62.3 \%$ at $-0.6 \mathrm{~V}$ vs. RHE (see also Figure S7).

The absence of any substantial improvement in the $\mathrm{FE}_{\mathrm{CO}}$ values at the lowest applied electrolysis potentials $\left(<-1.2 \mathrm{~V}\right.$ vs. RHE) can be rationalized by the onset of $\mathrm{CO}_{2}$ mass transfer limitations, where the $\mathrm{CO}_{2}$ concentration in the diffusion boundary layer is expected to drop down to zero as a result of increased $\mathrm{CO}_{2} \mathrm{RR}$ rates (partial current densities). Therefore, the continuous activation of the catalyst material under $\mathrm{CO}_{2}$ mass transport conditions does not lead to a further shift in the product distribution towards CO. The characteristics of pronounced hysteresis that can be seen at medium and low overpotentials (Figure 3a-e, Figure S7) are clearly indicative of the 'activation' of the Ag-NW catalyst towards $\mathrm{CO}$ formation, which is mediated by the applied electrochemical looping. This is demonstrated in the first experiment, as the co-electrolysis of water/ $\mathrm{CO}_{2}$ resulted in the desired deprotection (chemical cleaning) of the catalyst surface. It can be hypothesized that changes in the composition of the surface are responsible for the observed changes in the potential-dependent product distribution (see discussion on the XPS analysis below). A first control experiment proving that the improved FE values (Figure 3) indeed originate from an effective removal of the surfactants from the catalyst surface during the ec-1 treatment is shown in Figure S8. It compares the CO efficiencies of an Ag-NW catalyst before and after the ec-l treatment with the ones of an Ag-foil (GoodFellow, 99.95\%, $0.25 \mathrm{~mm}$ thickness) which serves as a model system for a surfactant-free Ag catalyst. As expected, the $\mathrm{CO}$ efficiencies do not change by the ec-l treatment in case of the Ag-foil catalyst. Further, we exclude severe structural or morphological changes of the Ag-NW catalyst in the course of the ec-1 treatment as origin of the observed catalyst activation (see combined SEM and TEM analysis in Figure S9). It should be noted that, based on our experimental results, it cannot be concluded on which active sites of the Ag-NWs the HER and the ec- $\mathrm{CO}_{2} \mathrm{RR}$ take place. Both experimental and theoretical studies on Ag single crystals strongly suggest, however, that defects, in particular steps and kink sites, are substantially more active towards CO formation than the planar (100) and (111) facets. ${ }^{10,68}$ 
One important aspect of this activation effect, discussed herein, is displayed in Figure 3f. In principle, the total (integrated) charge that is transferred at each electrolysis potential-derived from the respective $j$ versus $t$ (40 min.) plots - exponentially increases with the applied overpotential (Table S2a). However, when comparing the forward and backward runs, it becomes obvious that the total transferred charge for a given electrolysis potential does not substantially change during electrochemical looping. This implies that only the product distribution (ratio of $\mathrm{FE}_{\mathrm{CO}}$ and $\mathrm{FE}_{\mathrm{H} 2}$

values) is altered by this treatment, whereas the total current density normalized to the geometric surface area (total transferred charge) remains unaffected. This is an important distinction between the current study and previous studies on catalyst activation processes in which only a single electrocatalytic reaction needs to be considered (e.g., $\mathrm{ORR}^{40,49,55}$, $\mathrm{OER}^{49}$, or $\mathrm{HER}^{37}$ ) and where increased reaction rates directly correlate with an increase of the electrochemically active surface area (ECSA)..$^{53-54}$

In order to elaborate on which experimental factors contribute to the observed change in the product distribution (e.g. nature of the formed $\mathrm{CO}_{2} \mathrm{RR}$ reaction product, applied vertex potential $[\mathrm{E} \quad$ vertex $]$, current density [j], electrolysis time, total transferred charge [Q], etc.), an extra electrochemical looping experiment was carried out in an Ar-saturated $\left(\mathrm{CO}_{2}\right.$-free) $0.5 \mathrm{M} \mathrm{KHCO}_{3}$ electrolyte $(\mathrm{pH}=8.9)$ while applying the full range of electrolysis potentials $\left(\mathrm{E}_{\mathrm{vertex}}=-1.3 \mathrm{~V}\right.$ vs. RHE). This approach excludes $\mathrm{CO}$ as a reaction product and exclusively produces $\mathrm{H}_{2}$ during electrolysis. Note that bicarbonate can be neglected as a reactant when $\mathrm{Ag}$ is used as the catalyst. ${ }^{63}$ Figure $4 \mathrm{a}$ compares the total transferred charges of the chemical looping experiments carried out in the Ar- and the $\mathrm{CO}_{2}$ saturated electrolyte (Table S2a). The most obvious difference is in the total amount of transferred charges, which is substantially higher for the $\mathrm{CO}_{2}$-free case in which the HER is the only electrolytic reaction. These results suggest that the HER is not effectively hindered by the presence of the surfactants (chloride and PVP). Note that the expected exponential increase in the total transferred charge passes into a plateau regime at applied potentials that are more negative than $-1.1 \mathrm{~V}$ vs. RHE (Figure 4a). This particular feature originates from the partial blocking of the electrode surface by hydrogen bubbles, which appear at elevated current densities (surface area change under massive gas evolution; see Figure S10). 
It becomes obvious from Fig. 4a that the total transferred charges are substantially lower when $\mathrm{CO}$ is formed as one of the reaction products. This is likely owing to a high surface concentration of formed and temporarily adsorbed $* \mathrm{CO}$ (the asterisk represents an adsorption state), which therefore effectively sterically blocks those surface sites on the Ag-NW that are active towards the competing HER. It is clear that the chemisorbed ${ }^{*} \mathrm{CO}$ acts as an efficient 'suppressor' with regard to the HER. ${ }^{63}$ The binding strength of * $\mathrm{CO}$ to the $\mathrm{Ag}$ catalyst is generally considered to be relatively low (i.e. in comparison to $\mathrm{Cu})^{69-71}$, thereby rationalizing the easy release of the formed $* \mathrm{CO}$ from the catalyst surface into the electrolyte phase (Figure 5). However, the *CO binding to the Ag-NW surface seems sufficiently high to remove surfactants from the surface during the water $/ \mathrm{CO}_{2}$ co-electrolysis reaction, which can be considered to be the origin of the profound hysteresis effects observed in the $F E$ vs. $E$ plots (Figure 3). It can be hypothesized that the observed Ag-NW deprotection is based on the 'chemisorptive displacement' of the surfactants by the *CO . The temporary presence of chemisorbed * $\mathrm{CO}$ on the $\mathrm{Ag}$ catalyst surface has been previously demonstrated by operando vibrational (IR or Raman)

spectroscopy. ${ }^{72-74}$ The massive gas evolution (by $\mathrm{H}_{2} \quad$ and $\mathrm{CO}$ ) — which is in agreement with the water/ $\mathrm{CO}_{2}$ co-electrolysis at high current densities (Figure S10) — can be considered to be an additional beneficial effect and facilitates the convectional transport of the released PVP from the catalyst surface into the bulk of the electrolyte phase. This process therefore prevents the re-adsorption of the PVP on the catalyst surface. Possible surfactant re-adsorption phenomena have been identified by Oezaslan et al. ${ }^{54}$ as one possible drawback of the oxidative approach to PVP removal.

The chemical nature of the electrolysis product $\left(\mathrm{H}_{2}\right.$ or $\left.\mathrm{CO}\right)$ that is formed during the electrochemical looping clearly plays a vital role in the deprotection of the desired catalyst. This effect can be denoted as surfactant removal by 'chemical' cleaning. This has been demonstrated by additional experiments for $\mathrm{CO}_{2}$ electrolysis, which were performed at a constant electrolysis potential of $\mathrm{E}=-0.9 \mathrm{~V}$ vs. $\mathrm{RHE}$ using Ag-NW catalysts that had been subjected to a full chemical looping pretreatment $\left(\mathrm{E}_{\text {vertex }}=-1.3 \mathrm{~V}\right.$ vs. RHE) either in the $\mathrm{CO}_{2}$-saturated or the $\mathrm{CO}_{2}$-free (Ar-saturated) electrolyte. Figure $4 \mathrm{~b}$ illustrates the time-dependent evolution of the $\mathrm{FE}_{\mathrm{CO}}$ values of the electrolyses that were carried out in the $\mathrm{CO}_{2}$ saturated electrolyte following the ec-l treatments. 
For the purpose of comparison, the resulting $\mathrm{FE}_{\mathrm{CO}}$ values of the as-prepared samples are also provided. It is clear that maximal CO efficiency (close to $100 \%$ ) is most rapidly attained when pre-conditioning in the $\mathrm{CO}_{2}$-saturated electrolyte, whereas the one subjected to the chemical looping in the Ar-saturated electrolyte demonstrates only marginally improved $\mathrm{CO}$ efficiencies. This finding is striking, as substantially higher charges were transferred and higher current densities were applied during chemical looping in the Ar-saturated electrolyte $\left(Q_{\text {tot }}=895.9 \mathrm{C}, \mathrm{j}_{\max }=-85.6 \mathrm{~mA} \mathrm{~cm}^{-2}\right.$ at $\mathrm{E}=-1.3 \mathrm{~V}$ vs. RHE, see Table S2a) in comparison to the $\mathrm{CO}_{2}$-saturated electrolyte $\left(\mathrm{Q}_{\mathrm{tot}}=115.3 \mathrm{C}, \mathrm{j}_{\max }=-15.1 \mathrm{~mA}\right.$ $\mathrm{cm}^{-2}$ at $\mathrm{E}=-1.3 \mathrm{~V}$ vs. RHE). The total charge is obviously not the key parameter for the activation of the catalyst. Furthermore, the massive gas evolution alone does not seem to be sufficient for the deprotection of the Ag-NW catalyst (see also Figure S11).

As the total transferred charges were different in both electrochemical looping treatments (Ar- and $\mathrm{CO}_{2}$-saturated electrolytes, Figure $4 \mathrm{a}$ ) it is hard to compare them directly. We therefore applied two addition pretreatment techniques on the Ag-NW catalysts — based on galvanostatic electrolyses at $\mathrm{j}=$ $-3 \mathrm{~mA} \mathrm{~cm}^{-2}$ - in both $\mathrm{CO}_{2}$-saturated and $\mathrm{CO}_{2}$-free electrolytes. In these cases, the total transferred charge was normalized to $\mathrm{Q}_{\mathrm{tot}}=115.3 \mathrm{C}$, which allowed for a direct comparison to the electrochemical looping experiment performed in the $\mathrm{CO}_{2}$-containing electrolyte (Figure 4a). The corresponding $\mathrm{FE}_{\mathrm{CO}}$ data for the subsequent $\mathrm{CO}_{2}$ electrolysis reactions at $-0.9 \mathrm{~V}$ vs. RHE are included into the plot in Figure 4b. Again, pretreatment in the $\mathrm{CO}_{2}$-free electrolyte yields poor $\mathrm{FE}_{\mathrm{CO}}$ values in the actual $\mathrm{CO}_{2}$ electrolysis experiment. Interestingly, the electrochemical looping in the $\mathrm{CO}_{2}$-saturated electrolyte is superior to the galvanostatic pretreatment at $\mathrm{j}=-3 \mathrm{~mA} \mathrm{~cm} \mathrm{~cm}^{-2}$ that was carried out in the same electrolyte. Obviously, the applied electrolysis potential and the electrolysis time are important factors for the efficiency of surfactant removal (see also Figure S12 and S13, and discussion of the XPS data below). It can be assumed that, due to the increased CO partial current densities, the CO surface coverage is higher at lower vertex potentials thus also rationalizing the observed potential dependence of the hysteresis characteristics (Figure 3).

An extra electrolysis experiment was carried out using C-supported Ag-NWs as the catalyst in order to demonstrate that the electrochemical looping works when the NWs are embedded into a technical carbon matrix. The result of this ec-l experiment exhibits the desired trend of improved $\mathrm{FE}_{\mathrm{CO}}$ values in 
the corresponding backward run of the electrochemical looping (Figure 6), in which values of $\mathrm{FE}_{\mathrm{CO}}=$ $90.7 \%$ and $\mathrm{FE}_{\mathrm{CO}}=93.4 \%$ at $\mathrm{E}=-1.0 \mathrm{~V}$ and $-0.9 \mathrm{~V}$ vs. RHE were achieved. However, the HER is still dominating the product distribution at lower applied overpotentials in contrast to the non-supported Ag-NWs (see Figure 3e). This observation can be rationalized by an effect that is mediated by the high-surface area of the C-support, which is active towards the HER but not towards the $\mathrm{CO}_{2} \mathrm{RR}$. The increased $\mathrm{FE}_{\mathrm{H} 2}$ values at the lowest overpotentials (Figure 6) are therefore the result of a surface area effect of the component in the catalyst film, which is selective towards the HER (Vulcan and glassy carbon support electrode, see Figure S6d-f).

As the extended electrochemical looping $\left(\mathrm{E}_{\mathrm{vertex}}=-1.3 \mathrm{~V}\right)$ was identified as the most effective pretreatment for the deprotection of the catalyst, a full set of additional electrolysis experiments were performed using a single catalyst approach ${ }^{63}$ in which newly prepared and pre-conditioned catalyst (see Figure 2a-c and 3e) were used for $1 \mathrm{hr}$ long electrolysis experiments and applied potential. This approach guaranteed identical starting conditions for $\mathrm{CO}_{2}$ electrolysis and minimized time-dependent changes on the selectivity of the $\mathrm{CO}_{2} \mathrm{RR}$ products. Figure 7a represents the 'true' potential-dependent product distribution of the Ag-NW catalyst after the successful deprotection of the Ag-NWs. For comparison purposes, the corresponding $1 \mathrm{~h}$ lasting $e c-\mathrm{CO}_{2} \mathrm{RR}$ experiments of the as-prepared Ag-NW catalysts are also provided. CO efficiencies of $\sim 100 \%$ are obtained after the ec-1 pre-conditioning $\left(E_{\text {vertex }}=-1.3 \mathrm{~V}\right.$ vs. RHE) in the potential range between -1.0 and $-1.1 \mathrm{~V}$. These efficiencies are competitive in comparison to previously published data. ${ }^{20-21,63,75}$ Table S6 provides a comprehensive overview of the relevant benchmark studies that have used $\mathrm{Ag}$ as the $e c-\mathrm{CO}_{2} \mathrm{RR}$ catalyst material, while Figure $7 \mathrm{~b}$ demonstrates again that only the product selectivity is changed by the ec-l treatment, and not the overall reaction rate. The total (steady-state) current densities remain largely unaffected by electrochemical looping.

XPS analysis - Our analysis of the ec- $\mathrm{CO}_{2} \mathrm{RR}$ product distribution (Figures 3 and $7 \mathrm{a}$ ) clearly demonstrates an activation of the Ag-NW catalyst by the chemical looping, but lacks deeper mechanistic insights into the chemical origin of the observed improved CO selectivity. Therefore, complementary XPS experiments were performed to provide information on the compositional changes of the catalyst surface. Figure $8 \mathrm{a}-\mathrm{c}$ depicts spectra of the $\mathrm{Ag} 3 d, \mathrm{Cl} 2 p$, and $\mathrm{N} 1 s$ photo- 
emissions that are representative of the as-prepared Ag-NW catalyst prior to its deprotection. These results demonstrate that both chloride and PVP are present on the surface of the as-prepared Ag-NWs, as indicated in the schematics of Figure 9. The performed electrolysis experiments clearly show that the HER does not effectively contribute to the deprotection of the desired catalyst (Figure $4 \mathrm{~b}$ ).

Figure 8d-e displays the integrated intensities of the $\mathrm{N} 1 s$ and $\mathrm{Cl} 2 p$ emissions normalized to the one of the respective Ag3d emissions. These data can be used to assess the effectiveness of the surfactant removal depending on the particular pretreatment protocol that is applied. Note that the $\left(\mathrm{I}_{C l 2 p}: \mathrm{I}_{\mathrm{Ag} 3 d}\right)$ ratios are generally lower than the corresponding $\left(\mathrm{I}_{N l s}: \mathrm{I}_{\mathrm{Ag} 3 d}\right)$ values, irrespective of the applied pretreatment. One possible reason for this observation is that a layered structure of the surfactant shell was covering the Ag-NWs. Chloride is likely to be chemisorbed, and would therefore be in direct contact with the Ag-NW surface.

These halide anions are considered to play a crucial role in the initial nucleation stage of Ag-NW formation (self-seeding via $\mathrm{AgCl}$ nuclei) ${ }^{32}$. Furthermore, the (100) textured sidewalls of the Ag-NWs in particular exhibit a strong tendency towards specific chloride adsorption, which can result in a maximum (saturation) surface coverage of $\Theta=0.5 \mathrm{ML}$ (normalized to the number of surface atoms on the [100] surface) when an $\mathrm{Ag}(100)-\mathrm{c}(2 \times 2)-\mathrm{Cl}$ surface ad-layer is formed. ${ }^{76-78}$ The high molecular mass PVP polymer $\left(\mathrm{M}_{\mathrm{w}}=1300000 \mathrm{~g} \mathrm{~mol}^{-1}\right)$ presumably constitutes the outermost shell of the as deposited Ag-NW. A 'coiling' of the linear PVP around the Ag-NW is discussed in the literature, where the pyrrolidone acts as the anchor group of the polymer backbone to free metallic sites on the surface (Ag-O or Ag-N coordination) ${ }^{32}$. Considering the high molecular mass of the PVP, it is likely that hydrophobic effects lead to an enhanced PVP agglomeration on the Ag-NWs beyond monolayer coverages. This layered configuration of surfactants, as depicted in Figure 9 (left panel), could also contribute to the reduced intensity observed in the $\mathrm{Cl} 2 p$ emission of the chloride that accumulated at the 'buried' interface.

The electrochemical activation treatments applied to the Ag-NW catalysts exhibit strong variations in the PVP removal efficiency. The treatments in which $\mathrm{H}_{2}$ was the exclusive electrolysis product (protocols 2 and 3 in Figure 8d) were less effective, while those using postsynthesis deprotection approaches involving the formation of $\mathrm{CO}$ (protocol 4 and 5 in Figure $8 \mathrm{~d}$ ) were more effective. The 
optimal PVP removal characteristics that were observed for the electrochemical looping approach $\left(E_{\text {vertex }}=-1.3 \mathrm{~V}\right.$ vs. RHE) are in full agreement with our electrolysis data (Figure $4 \mathrm{~b}$ and $7 \mathrm{a}$ ). The XPS results also confirm that the PVP (and its removal) is the main origin for the observed hysteresis effects in the product distribution (Figure 3).

Interestingly, all pretreatments that were applied herein, led to the near-complete removal of the chemisorbed chloride (Figure 8e). Origin of the chloride removal is the potential-dependent electrostatic repulsion of the chloride anions at the negatively polarized electrode surface.

From these observations it can safely be concluded that it is the remaining PVP that disturbs the ec$\mathrm{CO}_{2} \mathrm{RR}$ rather than the chemisorbed chloride. Our analyses were further complemented by an extra XPS inspection of the catalyst films subjected to the systematic electrochemical looping experiments presented in Figure 3a-d. The results of this analysis are depicted in Figure 8f-g and clearly demonstrate that the vertex potential $\mathrm{E}_{\text {vertex }}$ and the width of the potential window that were applied to the catalysts in the electrochemical looping are necessary for the effectiveness of the surfactant removal. The surface concentration of adsorbed chloride could be reduced to the minimum possible quantity when vertex potentials of $E_{\text {vertex }}=-1.1 \mathrm{~V}$ vs. RHE were applied, whereas the PVP surface coverage continued to decrease to an applied vertex potential of $E_{\text {vertex }}=-1.3 \mathrm{~V}$ vs. RHE. Our XPS results are also in full agreement with the working hypothesis made on the basis of the electrolysis data presented in Figure 3, and confirm that compositional changes at the catalyst surface are the origin of the hysteresis features observed in the electrochemical analysis (see also Figure S15). Complementary ${ }^{1} \mathrm{H}-\mathrm{NMR}$ measurements suggest that the PVP is removed structurally intact from the Ag surface. There are no PVP degradation products observed in the electrolyte after the electrolysis.

\section{Conclusions and outlook}

Here, we demonstrate that the presence of surfactants (e.g. chloride and in particular PVP) on the surface of the colloidal silver catalyst negatively impacts the $e c-\mathrm{CO}_{2} \mathrm{RR}$ selectivity and instead favours the HER in electrolysis reactions carried out in $\mathrm{CO}_{2}$-saturated aqueous $0.5 \mathrm{M}$ bicarbonate electrolytes. 
The present work clearly demonstrates the importance of complete surfactant removal for the catalyst performance evaluation which might otherwise be superimposed by 'transient artefacts', in particular in the initial stage of electrolysis (time scale of hours).

Electrochemical looping — a sequence of potentiostatic electrolysis experiments with defined starting, vertex, and ending potentials — has been demonstrated to be highly effective in the deprotection of catalysts, provided that $\mathrm{CO}$ is formed as the main electrolysis product. The chemical nature of the reaction product formed during electrolysis is found to be vital to the effectiveness of the activation of the catalysts via surfactant removal. An extended potential window in the electrochemical looping pretreatment, spanning from $E_{\text {start }}=-0.6 \mathrm{~V}$ vs. RHE to $E_{\text {vertex }}=-1.3 \mathrm{~V}$ vs. RHE, yields substantially improved $\mathrm{CO}$ efficiencies, which attained $\mathrm{FE}_{\mathrm{CO}}=100 \%$ at $-1.0 \mathrm{~V}\left(\mathrm{j}_{\mathrm{CO}}=-5.8 \mathrm{~mA} \mathrm{~cm}^{-2}\right)$ and $-1.1 \mathrm{~V}$ vs. RHE $\left(\mathrm{j}_{\mathrm{CO}}=-6.5 \mathrm{~mA} \mathrm{~cm}{ }^{-2}\right)$. This improvement in the product selectivity relative to the as-prepared $\mathrm{Ag}-$ NWs is in agreement with the observed decrease in the normalized PVP surface concentration. This catalyst deprotection protocol is also transferable to C-supported Ag-NW catalyst systems.

Our future research will address the application of these electrochemically activated Ag-NW catalysts in flow-cell electrolyser systems in detail in order to demonstrate the importance of the environment (gaseous versus aqueous/liquid) for surfactant removal under operando experimental conditions.

\section{Additional information}

Supplementary information is available in "Supplementary Information" file.

\section{Corresponding Authors}

*Email: peter.broekmann@ddcb.unibe.ch; yuhui.hou@dcb.unibe.ch

\section{Declaration of competing interest}

There is no conflict of interests.

\section{Acknowledgments}

The financial support by the CTI Swiss Competence Center for Energy Research (SCCER Heat and Electricity Storage) is gratefully acknowledged. PB acknowledges the financial support by the Swiss National Science Foundation (SNSF) via the project No. 200020_172507. HH, ML, YK, and CS 
acknowledge the financial support by the Chinese Scholarship Council (CSC). YH acknowledges the financial support by the University of Bern.

\section{References}

1. Change is in the air. Nat. Catal. 2018, 1 (2), 93-93.

2. Jhong, H.-R. M.; Ma, S.; Kenis, P. J. A., Electrochemical Conversion of $\mathrm{CO}_{2}$ to Useful Chemicals: Current Status, Remaining Challenges, and Future Opportunities. Curr. Opin. Chem. Eng. 2013, 2 (2), 191-199.

3. Jones, J.-P.; Prakash, G. K. S.; Olah, G. A., Electrochemical $\mathrm{CO}_{2}$ Reduction: Recent Advances and Current Trends. Isr. J. Chem. 2014, 54 (10), 1451-1466.

4. Whipple, D. T.; Kenis, P. J. A., Prospects of $\mathrm{CO}_{2}$ Utilization via Direct Heterogeneous Electrochemical Reduction. J. Phys. Chem. Lett. 2010, 1 (24), 3451-3458.

5. Durst, J.; Rudnev, A.; Dutta, A.; Fu, Y.; Herranz, J.; Kaliginedi, V.; Kuzume, A.; Permyakova, A. A.; Paratcha, Y.; Broekmann, P.; Schmidt, T. J., Electrochemical $\mathrm{CO}_{2}$ Reduction - A Critical View on Fundamentals, Materials and Applications. CHIMIA 2015, 69 (12), 769-776.

6. Samavati, M.; Santarelli, M.; Martin, A.; Nemanova, V., Production of Synthetic FischerTropsch Diesel from Renewables: Thermoeconomic and Environmental Analysis. Energy \& Fuels 2018, 32 (2), 1744-1753.

7. Haas, T.; Krause, R.; Weber, R.; Demler, M.; Schmid, G., Technical photosynthesis involving $\mathrm{CO}_{2}$ electrolysis and fermentation. Nat. Catal. 2018, 1 (1), 32-39.

8. Hori, Y.; Wakebe, H.; Tsukamoto, T.; Koga, O., Electrocatalytic Process of CO Selectivity in Electrochemical Reduction of $\mathrm{CO}_{2}$ at Metal Electrodes in Aqueous Media. Electrochim. Acta 1994, 39 (11-12), 1833-1839.

9. Hori, Y.; Kikuchi, K.; Suzuki, S., Production of $\mathrm{CO}$ and $\mathrm{CH}_{4}$ in Electrochemical Reduction of $\mathrm{CO}_{2}$ at Metal-Electrodes in Aqueous Hydrogencarbonate Solution Chem. Lett. 1985, (11), 1695-1698.

10. Hoshi, N.; Kato, M.; Hori, Y., Electrochemical reduction of $\mathrm{CO}_{2}$ on single crystal electrodes of silver $\operatorname{Ag}(111), \operatorname{Ag}(100)$ and $\operatorname{Ag}(110)$. J. Electroanal. Chem. 1997, 440 (1-2), 283-286.

11. Boles, M. A.; Ling, D.; Hyeon, T.; Talapin, D. V., The surface science of nanocrystals. Nat. Mater. 2016, 15 (2), 141-153.

12. Back, S.; Yeom, M. S.; Jung, Y., Active Sites of Au and Ag Nanoparticle Catalysts for $\mathrm{CO}_{2}$ Electroreduction to CO. ACS Catal. 2015, 5 (9), 5089-5096.

13. Gabardo, C. M.; Seifitokaldani, A.; Edwards, J. P.; Dinh, C.-T.; Burdyny, T.; Kibria, M. G.; O’Brien, C. P.; Sargent, E. H.; Sinton, D., Combined high alkalinity and pressurization enable efficient $\mathrm{CO}_{2}$ electroreduction to CO. Energy Environ. Sci. 2018, 11 (9), 2531-2539.

14. Salvatore, D. A.; Weekes, D. M.; He, J.; Dettelbach, K. E.; Li, Y. C.; Mallouk, T. E.; Berlinguette, C. P., Electrolysis of Gaseous $\mathrm{CO}_{2}$ to $\mathrm{CO}$ in a Flow Cell with a Bipolar Membrane. ACS Energy Lett. 2018, 3 (1), 149-154.

15. Larrazábal, G. O.; Strøm-Hansen, P.; Heli, J. P.; Zeiter, K.; Therkildsen, K. T.; Chorkendorff, I.; Seger, B., Analysis of Mass Flows and Membrane Cross-over in $\mathrm{CO}_{2}$ Reduction at High Current Densities in an MEA-Type Electrolyzer. ACS Appl. Mater. Interfaces 2019, $11(44), 41281$ 41288. 
16. Liu, Z.; Yang, H.; Kutz, R.; Masel, R. I., $\mathrm{CO}_{2}$ Electrolysis to $\mathrm{CO}$ and $\mathrm{O}_{2}$ at High Selectivity, Stability and Efficiency Using Sustainion Membranes. J. Electrochem. Soc. 2018, 165 (15), J3371-J3377.

17. Verma, S.; Kim, B.; Jhong, H.-R. M.; Ma, S.; Kenis, P. J. A., A Gross-Margin Model for Defining Technoeconomic Benchmarks in the Electroreduction of $\mathrm{CO}_{2}$. ChemSusChem 2016, 9 (15), 1972-1979.

18. Martín, A. J.; Larrazábal, G. O.; Pérez-Ramírez, J., Towards sustainable fuels and chemicals through the electrochemical reduction of $\mathrm{CO}_{2}$ : lessons from water electrolysis. Green Chem. 2015, 17 (12), 5114-5130.

19. Kan, C. X.; Zhu, J. J.; Zhu, X. G., Silver nanostructures with well-controlled shapes: synthesis, characterization and growth mechanisms. J. Phys. D 2008, 41 (15).

20. Liu, S.; Sun, C.; Xiao, J.; Luo, J.-L., Unraveling Structure Sensitivity in $\mathrm{CO}_{2}$ Electroreduction to Near-Unity CO on Silver Nanocubes. ACS Catal. 2020, 3158-3163.

21. Liu, S.; Tao, H.; Zeng, L.; Liu, Q.; Xu, Z.; Liu, Q.; Luo, J.-L., Shape-Dependent Electrocatalytic Reduction of $\mathrm{CO}_{2}$ to $\mathrm{CO}$ on Triangular Silver Nanoplates. J. Am. Chem. Soc. 2017, 139 (6), 2160-2163.

22. Zhang, S. H.; Jiang, Z. Y.; Xie, Z. X.; Xu, X.; Huang, R. B.; Zheng, L. S., Growth of silver nanowires from solutions: A cyclic penta-twinned-crystal growth mechanism. J. Phys. Chem. B 2005, 109 (19), 9416-9421.

23. Xi, W.; Ma, R.; Wang, H.; Gao, Z.; Zhang, W.; Zhao, Y., Ultrathin Ag Nanowires Electrode for Electrochemical Syngas Production from Carbon Dioxide. ACS Sustainable Chem. Eng. 2018, 6 (6), 7687-7694.

24. Sun, Y. G.; Yin, Y. D.; Mayers, B. T.; Herricks, T.; Xia, Y. N., Uniform silver nanowires synthesis by reducing $\mathrm{AgNO}_{3}$ with ethylene glycol in the presence of seeds and poly(vinyl pyrrolidone). Chem. Mater. 2002, 14 (11), 4736-4745.

25. Li, X.; Wang, L.; Yan, G., Review: Recent research progress on preparation of silver nanowires by soft solution method and their applications. Cryst. Res. Technol. 2011, 46 (5), 427-438.

26. Li, B.; Ye, S.; Stewart, I. E.; Alvarez, S.; Wiley, B. J., Synthesis and Purification of Silver Nanowires To Make Conducting Films with a Transmittance of 99\%. Nano Lett. 2015, 15 (10), 6722-6726.

27. Jiu, J.; Araki, T.; Wang, J.; Nogi, M.; Sugahara, T.; Nagao, S.; Koga, H.; Suganuma, K.; Nakazawa, E.; Hara, M.; Uchida, H.; Shinozaki, K., Facile synthesis of very-long silver nanowires for transparent electrodes. J. Mater. Chem. A 2014, 2 (18), 6326-6330.

28. Xia, Y.; Xiong, Y.; Lim, B.; Skrabalak, S. E., Shape-Controlled Synthesis of Metal Nanocrystals: Simple Chemistry Meets Complex Physics? Angew. Chem. Int. Ed. 2009, 48 (1), 60-103.

29. Coskun, S.; Aksoy, B.; Unalan, H. E., Polyol Synthesis of Silver Nanowires: An Extensive Parametric Study. Cryst. Growth Des. 2011, 11 (11), 4963-4969.

30. Gao, Y.; Jiang, P.; Song, L.; Liu, L. F.; Yan, X. Q.; Zhou, Z. Q.; Liu, D. F.; Wang, J. X.; Yuan, H. J.; Zhang, Z. X.; Zhao, X. W.; Dou, X. Y.; Zhou, W. Y.; Wang, G.; Xie, S. S., Growth mechanism of silver nanowires synthesized by polyvinylpyrrolidone-assisted polyol reduction. J. Phys. D: Appl. Phys. 2005, 38 (7), 1061-1067.

31. Kim, M. J.; Alvarez, S.; Chen, Z.; Fichthorn, K. A.; Wiley, B. J., Single-Crystal Electrochemistry Reveals Why Metal Nanowires Grow. J. Am. Chem. Soc. 2018, 140 (44), 14740-14746. 
32. Sun, Y.; Xia, Y., Large-Scale Synthesis of Uniform Silver Nanowires Through a Soft, SelfSeeding, Polyol Process. Adv. Mater. 2002, 14 (11), 833-837.

33. Sun, D.; Xu, X.; Qin, Y.; Jiang, S. P.; Shao, Z., Rational Design of Ag-Based Catalysts for the Electrochemical $\mathrm{CO}_{2}$ Reduction to CO: A Review. ChemSusChem 2020, 13 (1), 39-58.

34. Mourdikoudis, S.; Liz-Marzán, L. M., Oleylamine in Nanoparticle Synthesis. Chem. Mater. 2013, 25 (9), 1465-1476.

35. Yang, H.-J.; He, S.-Y.; Tuan, H.-Y., Self-Seeded Growth of Five-Fold Twinned Copper Nanowires: Mechanistic Study, Characterization, and SERS Applications. Langmuir 2014, 30 (2), 602-610.

36. Jin, M.; He, G.; Zhang, H.; Zeng, J.; Xie, Z.; Xia, Y., Shape-Controlled Synthesis of Copper Nanocrystals in an Aqueous Solution with Glucose as a Reducing Agent and Hexadecylamine as a Capping Agent. Angew. Chem. Int. Ed. 2011, 50 (45), 10560-10564.

37. Ung, D.; Cossairt, B. M., Effect of Surface Ligands on CoP for the Hydrogen Evolution Reaction. ACS Appl. Energy Mater. 2019, 2 (3), 1642-1645.

38. Luo, M.; Hong, Y.; Yao, W.; Huang, C.; Xu, Q.; Wu, Q., Facile removal of polyvinylpyrrolidone (PVP) adsorbates from Pt alloy nanoparticles. J. Mater. Chem. A 2015, 3 (6), 2770-2775.

39. Lopez-Sanchez, J. A.; Dimitratos, N.; Hammond, C.; Brett, G. L.; Kesavan, L.; White, S.; Miedziak, P.; Tiruvalam, R.; Jenkins, R. L.; Carley, A. F.; Knight, D.; Kiely, C. J.; Hutchings, G. J., Facile removal of stabilizer-ligands from supported gold nanoparticles. Nat. Chem. 2011, 3 (7), 551-556.

40. Naresh, N.; Wasim, F. G. S.; Ladewig, B. P.; Neergat, M., Removal of surfactant and capping agent from Pd nanocubes (Pd-NCs) using tert-butylamine: its effect on electrochemical characteristics. J. Mater. Chem. A 2013, 1 (30), 8553-8559.

41. Cargnello, M.; Chen, C.; Diroll, B. T.; Doan-Nguyen, V. V. T.; Gorte, R. J.; Murray, C. B., Efficient Removal of Organic Ligands from Supported Nanocrystals by Fast Thermal Annealing Enables Catalytic Studies on Well-Defined Active Phases. J. Am. Chem. Soc. 2015, 137 (21), 6906-6911.

42. Rioux, R. M.; Song, H.; Grass, M.; Habas, S.; Niesz, K.; Hoefelmeyer, J. D.; Yang, P.; Somorjai, G. A., Monodisperse platinum nanoparticles of well-defined shape: synthesise characterization, catalytic properties and future prospects. Top. Catal. 2006, 39 (3-4), 167-174.

43. Hou, Y.; Bolat, S.; Bornet, A.; Romanyuk, Y. E.; Guo, H.; Moreno-García, P.; Zelocualtecatl Montiel, I.; Lai, Z.; Müller, U.; Grozovski, V.; Broekmann, P., Photonic Curing: Activation and Stabilization of Metal Membrane Catalysts (MMCs) for the Electrochemical Reduction of $\mathrm{CO}_{2}$. ACS Catal. 2019, 9 (10), 9518-9529.

44. Bartholomew, C. H., Mechanisms of catalyst deactivation. Appl. Catal. A 2001, 212 (1-2), 1760 .

45. Shaw, S.; Tian, X. C.; Silva, T. F.; Bobbitt, J. M.; Naab, F.; Rodrigues, C. L.; Smith, E. A.; Cademartiri, L., Selective Removal of Ligands from Colloidal Nanocrystal Assemblies with Non-Oxidizing He Plasmas. Chem. Mater. 2018, 30 (17), 5961-5967.

46. Aliaga, C.; Park, J. Y.; Yamada, Y.; Lee, H. S.; Tsung, C.-K.; Yang, P.; Somorjai, G. A., Sum Frequency Generation and Catalytic Reaction Studies of the Removal of Organic Capping Agents from Pt Nanoparticles by UV-Ozone Treatment. J. Phys. Chem. C 2009, 113 (15), 6150-6155.

47. Rosen, E. L.; Buonsanti, R.; Llordes, A.; Sawvel, A. M.; Milliron, D. J.; Helms, B. A., Exceptionally Mild Reactive Stripping of Native Ligands from Nanocrystal Surfaces by Using Meerwein's Salt. Angew. Chem. Int. Ed. 2012, 51 (3), 684-689. 
48. Doris, S. E.; Lynch, J. J.; Li, C. Y.; Wills, A. W.; Urban, J. J.; Helms, B. A., Mechanistic Insight into the Formation of Cationic Naked Nanocrystals Generated under Equilibrium Control. J. Am. Chem. Soc. 2014, 136 (44), 15702-15710.

49. Nelson, A.; Zong, Y.; Fritz, K. E.; Suntivich, J.; Robinson, R. D., Assessment of Soft Ligand Removal Strategies: Alkylation as a Promising Alternative to High-Temperature Treatments for Colloidal Nanoparticle Surfaces. ACS Mater. Lett. 2019, 1 (1), 177-184.

50. Menard, L. D.; Xu, F. T.; Nuzzo, R. G.; Yang, J. C., Preparation of TiO ${ }_{2}$-supported Au nanoparticle catalysts from a Au-13 cluster precursor: Ligand removal using ozone exposure versus a rapid thermal treatment. J. Catal. 2006, 243 (1), 64-73.

51. Ansar, S. M.; Perera, G. S.; Ameer, F. S.; Zou, S. L.; Pittman, C. U.; Zhang, D. M., Desulfurization of Mercaptobenzimidazole and Thioguanine on Gold Nanoparticles Using Sodium Borohydride in Water at Room Temperature. J. Phys. Chem. C 2013, 117 (26), $13722-$ 13729.

52. Hasché, F.; Oezaslan, M.; Strasser, P., In Situ Observation of the Thermally Induced Growth of Platinum-Nanoparticle Catalysts Using High-Temperature X-ray Diffraction. ChemPhysChem 2012, 13 (3), 828-834.

53. Li, D. G.; Wang, C.; Tripkovic, D.; Sun, S. H.; Markovic, N. M.; Stamenkovic, V. R., Surfactant Removal for Colloidal Nanoparticles from Solution Synthesis: The Effect on Catalytic Performance. ACS Catal. 2012, 2 (7), 1358-1362.

54. Safo, I. A.; Oezaslan, M., Electrochemical Cleaning of Polyvinylpyrrolidone-capped Pt Nanocubes for the Oxygen Reduction Reaction. Electrochim. Acta 2017, 241, 544-552.

55. Safo, I. A.; Dosche, C.; Özaslan, M., Effects of Capping Agents on the Oxygen Reduction Reaction Activity and Shape Stability of Pt Nanocubes. ChemPhysChem 2019, 20 (22), 30103023.

56. Bong, S.; Jang, B.; Han, D.; Piao, Y., Effective Electrochemical Activation of Oleate-ResidueFouled Pt Nanoparticle Catalysts for Methanol and Formic Acid Oxidation. ACS Omega 2019, 4 (23), 20330-20334.

57. Sun, Y. G.; Gates, B.; Mayers, B.; Xia, Y. N., Crystalline silver nanowires by soft solution processing. Nano Lett. 2002, 2 (2), 165-168.

58. Sun, Y. G.; Mayers, B.; Herricks, T.; Xia, Y. N., Polyol synthesis of uniform silver nanowires: A plausible growth mechanism and the supporting evidence. Nano Lett. 2003, 3 (7), 955-960.

59. Hori, Y., Electrochemical $\mathrm{CO}_{2}$ Reduction on Metal Electrodes. In Modern Aspects of Electrochemistry, Springer: New York, 2008; pp 89-189.

60. Dutta, A.; Rahaman, M.; Luedi, N. C.; Mohos, M.; Broekmann, P., Morphology Matters: Tuning the Product Distribution of $\mathrm{CO}_{2}$ Electroreduction on Oxide-Derived Cu Foam Catalysts. ACS Catal. 2016, 6 (6), 3804-3814.

61. Dutta, A.; Rahaman, M.; Mohos, M.; Zanetti, A.; Broekmann, P., Electrochemical $\mathrm{CO}_{2}$ Conversion Using Skeleton (Sponge) Type of Cu Catalysts. ACS Catal. 2017, 7 (8), 5431-5437.

62. Rahaman, M.; Dutta, A.; Broekmann, P., Size-Dependent Activity of Palladium Nanoparticles: Efficient Conversion of $\mathrm{CO}_{2}$ into Formate at Low Overpotentials. ChemSusChem 2017, 10 (8), 1733-1741.

63. Dutta, A.; Morstein, C. E.; Rahaman, M.; Cedeño López, A.; Broekmann, P., Beyond Copper in $\mathrm{CO}_{2}$ Electrolysis: Effective Hydrocarbon Production on Silver-Nanofoam Catalysts. ACS Catal. 2018, 8357-8368. 
64. da Silva, R. R.; Yang, M.; Choi, S.-I.; Chi, M.; Luo, M.; Zhang, C.; Li, Z.-Y.; Camargo, P. H. C.; Ribeiro, S. J. L.; Xia, Y., Facile Synthesis of Sub-20 nm Silver Nanowires through a Bromide-Mediated Polyol Method. ACS Nano 2016, 10 (8), 7892-7900.

65. Niu, Z.; Cui, F.; Kuttner, E.; Xie, C.; Chen, H.; Sun, Y.; Dehestani, A.; Schierle-Arndt, K.; Yang, P., Synthesis of Silver Nanowires with Reduced Diameters Using Benzoin-Derived Radicals to Make Transparent Conductors with High Transparency and Low Haze. Nano Lett. 2018, $18(8), 5329-5334$.

66. Jiang, P.; Li, S.-Y.; Xie, S.-S.; Gao, Y.; Song, L., Machinable Long PVP-Stabilized Silver Nanowires. Chem. Eur. J. 2004, 10 (19), 4817-4821.

67. Li, Y.; Cui, F.; Ross, M. B.; Kim, D.; Sun, Y.; Yang, P., Structure-Sensitive $\mathrm{CO}_{2}$ Electroreduction to Hydrocarbons on Ultrathin 5-fold Twinned Copper Nanowires. Nano Lett. 2017, 17 (2), 1312-1317.

68. Clark, E. L.; Ringe, S.; Tang, M.; Walton, A.; Hahn, C.; Jaramillo, T. F.; Chan, K.; Bell, A. T., Influence of Atomic Surface Structure on the Activity of Ag for the Electrochemical Reduction of $\mathrm{CO}_{2}$ to CO. ACS Catal. 2019, 9 (5), 4006-4014.

69. Kuhl, K. P.; Hatsukade, T.; Cave, E. R.; Abram, D. N.; Kibsgaard, J.; Jaramillo, T. F., Electrocatalytic Conversion of Carbon Dioxide to Methane and Methanol on Transition Metal Surfaces. J. Am. Chem. Soc. 2014, 136 (40), 14107-14113.

70. Liu, X. Y.; Xiao, J. P.; Peng, H. J.; Hong, X.; Chan, K.; Norskov, J. K., Understanding trends in electrochemical carbon dioxide reduction rates. Nat. Commun. 2017, 8 .

71. Hammer, B.; Morikawa, Y.; Nørskov, J. K., CO Chemisorption at Metal Surfaces and Overlayers. Phys. Rev. Lett. 1996, 76 (12), 2141-2144.

72. Zhu, S.; Li, T.; Cai, W.-B.; Shao, M., $\mathrm{CO}_{2}$ Electrochemical Reduction As Probed through Infrared Spectroscopy. ACS Energy Lett. 2019, 4 (3), 682-689.

73. Schmitt, K. G.; Gewirth, A. A., In Situ Surface-Enhanced Raman Spectroscopy of the Electrochemical Reduction of Carbon Dioxide on Silver with 3,5-Diamino-1,2,4-Triazole. $J$. Phys. Chem. C 2014, 118 (31), 17567-17576.

74. Ichinohe, Y.; Wadayama, T.; Hatta, A., Electrochemical reduction of $\mathrm{CO}$ on silver as probed by surface-enhanced Raman scattering. J. Raman Spectrosc. 1995, 26 (5), 335-340.

75. Liu, S.; Wang, X.-Z.; Tao, H.; Li, T.; Liu, Q.; Xu, Z.; Fu, X.-Z.; Luo, J.-L., Ultrathin 5-Fold Twinned Sub-25 nm Silver Nanowires Enable Highly Selective Electroreduction of $\mathrm{CO}_{2}$ to $\mathrm{CO}$. Nano Energy 2018, 45.

76. Fu, H.; Jia, L. L.; Wang, W. N.; Fan, K. N., The first-principle study on chlorine-modified silver surfaces. Surf. Sci. 2005, 584 (2-3), 187-198.

77. Bowker, M.; Waugh, K. C.; Wolfindale, B.; Lamble, G.; King, D. A., THE ADSORPTION OF CHLORINE AND CHLORIDATION OF AG(100). Surf. Sci. 1987, 179 (2-3), 254-266.

78. Lamble, G. M.; Brooks, R. S.; Campuzano, J. C.; King, D. A.; Norman, D., STRUCTURE OF THE C(2X2) COVERAGE OF CL ON AG(100) - A CONTROVERSY RESOLVED BY SURFACE EXTENDED X-RAY-ABSORPTION FINE-STRUCTURE SPECTROSCOPY. Phys. Rev. B 1987, 36 (3), 1796-1798. 


\section{Figures}

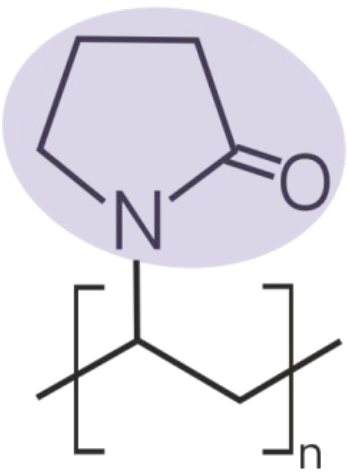

Figure 1. Polyvinylpyrrolidone (PVP) used as capping agent for the Ag-NW synthesis. The pyrrolidone functionality attached to the linear aliphatic backbone is highlighted purple. 

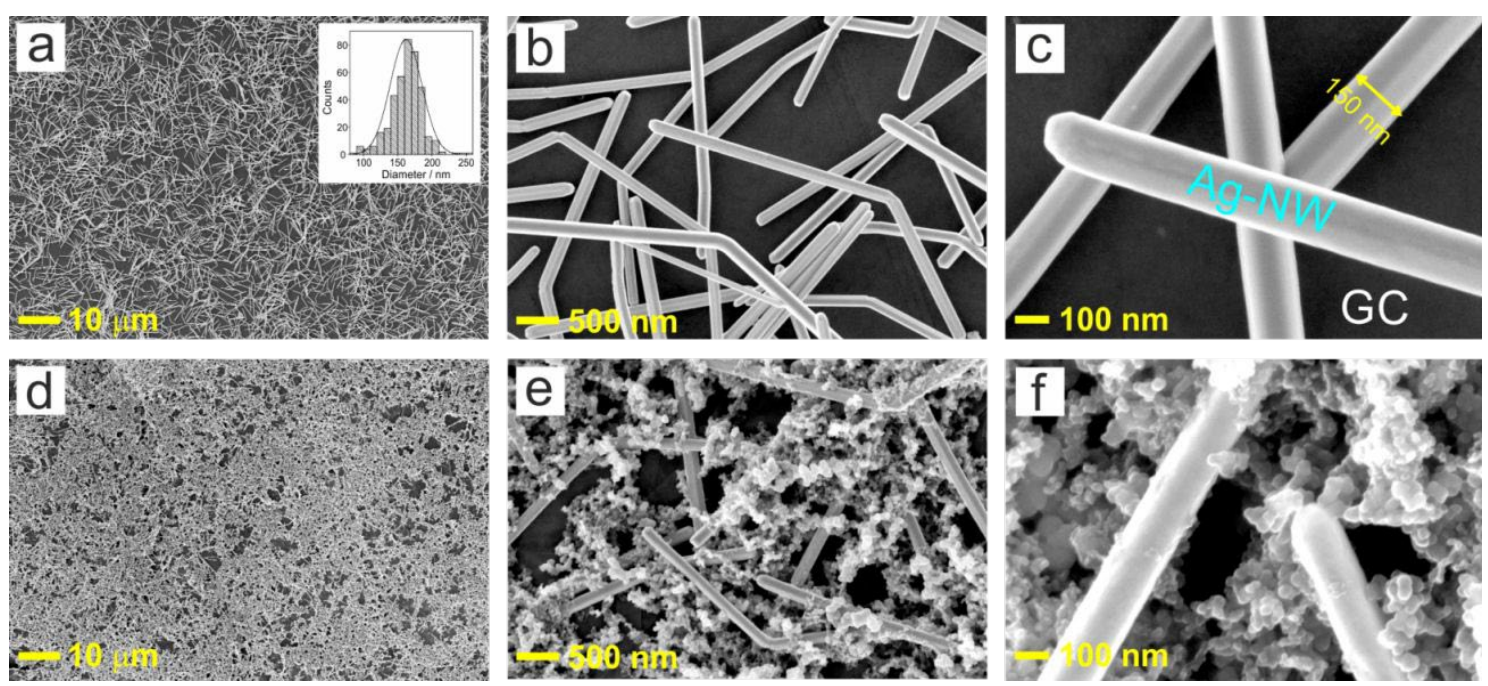

Figure 2. a) - c) Top-down SEM images of the Ag-NW film drop-casted on the glassy carbon (GC) support electrode, the inset in panel a shows a histogram representing the thickness distribution of the Ag-NWs;

d) - f) Corresponding SEM images of the C-supported (Vulcan XC 72R) Ag-NWs drop-casted on the GC support electrode (for details see Experimental section). 

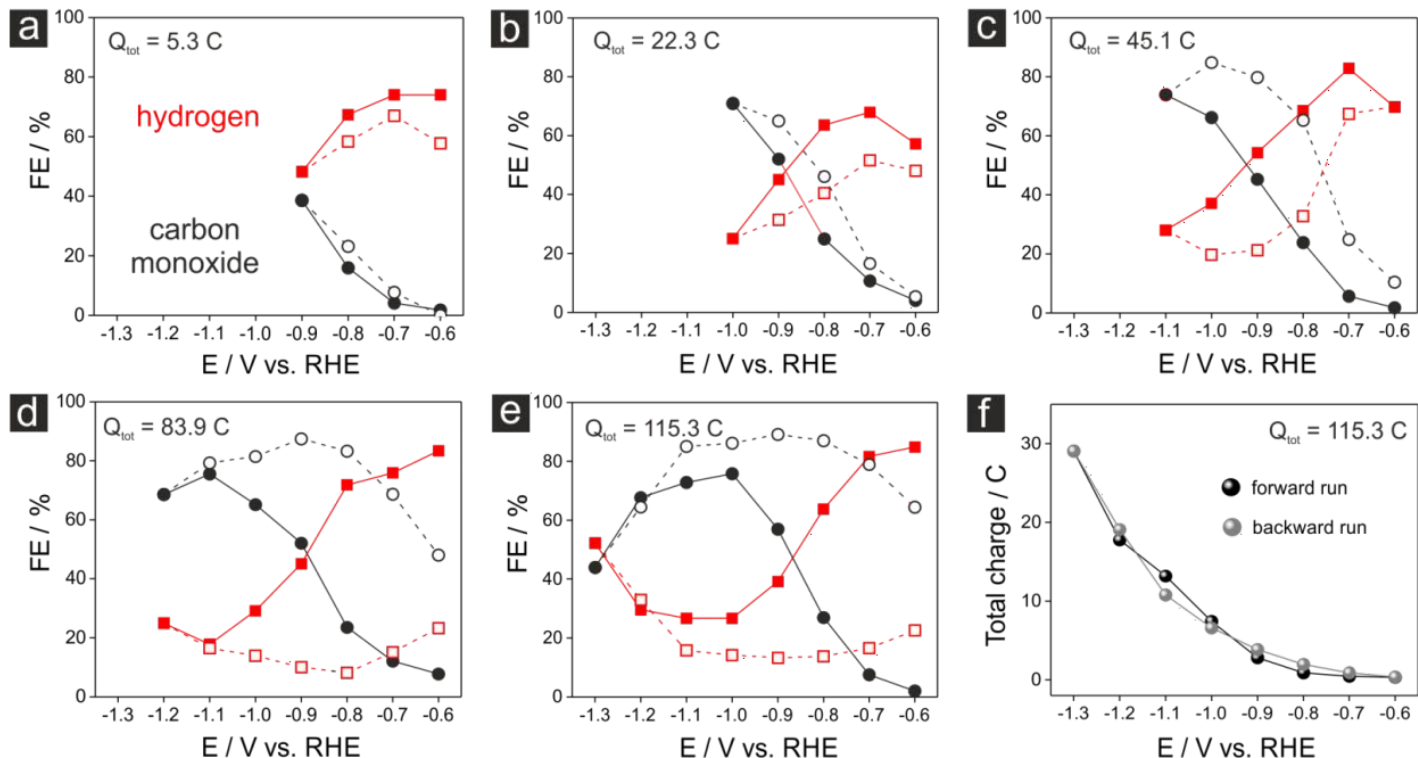

Figure 3. a) - e) Hysteresis effects appearing in the forward and backward runs of the electrochemical looping experiments (40 min. duration at each potential) carried out over Ag-NW catalysts (see Figure 2a-c) in $\mathrm{CO}_{2}$-saturated $0.5 \mathrm{M} \mathrm{KHCO}_{3}$ (single catalyst approach), the total cathodic charges transferred during the 'electrochemical looping' are indicated; f) Graph showing the total integrated charge corresponding to the electrolysis experiments shown in panel e. 


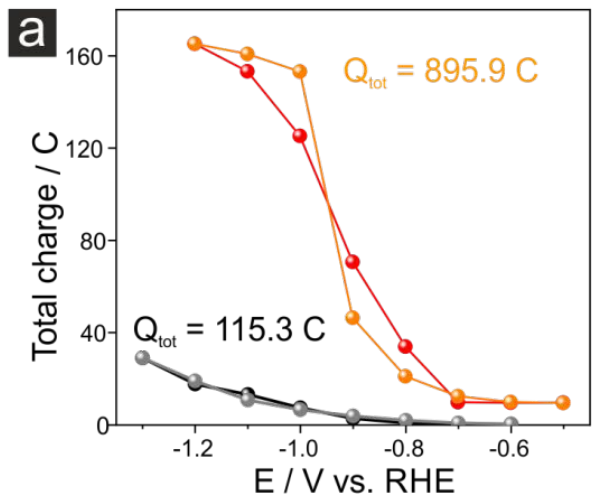

forward run ( $\mathrm{CO}_{2}$-sat.) forward run (Ar-sat.) backward run ( $\mathrm{CO}_{2}$-sat.) $\bigcirc$ backward run (Ar-sat.)

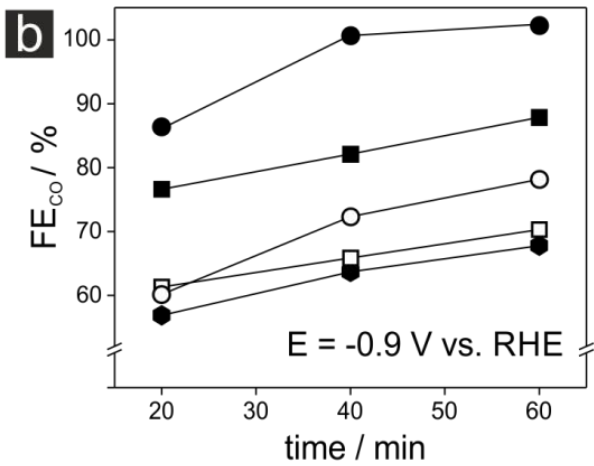

as prepared

- galvanostatic $\left(-3 \mathrm{~mA} \mathrm{~cm}^{-2}\right) ; \mathrm{CO}_{2}$-sat. Oe-looping; Ar-sat. $\square$ galvanostatic $\left(-3 \mathrm{~mA} \mathrm{~cm}^{-2}\right)$; Ar-sat. ec-looping; $\mathrm{CO}_{2}$-sat.

Figure 4: a) Integrated cathodic charges of potentiostatic electrolysis reactions carried out in Ar- and $\mathrm{CO}_{2}$-saturated $0.5 \mathrm{M} \mathrm{KHCO}_{3}$ electrolytes (electrochemical looping); b) Time-resolved FECO values derived from electrolysis reactions carried out at $-0.9 \mathrm{~V}$ vs RHE after applying various activation protocols (for details see text). 
Figure 5. Reaction pathway of $\mathrm{CO}_{2}$ conversion into $\mathrm{CO}$ on $\mathrm{Ag}$ catalysts; the strong suppressing action of the chemisorbed $\mathrm{CO}$ with regard to the HER is highlighted. 
Figure 6. Activation of C-supported Ag-NW catalysts (see Figure 2d-f).

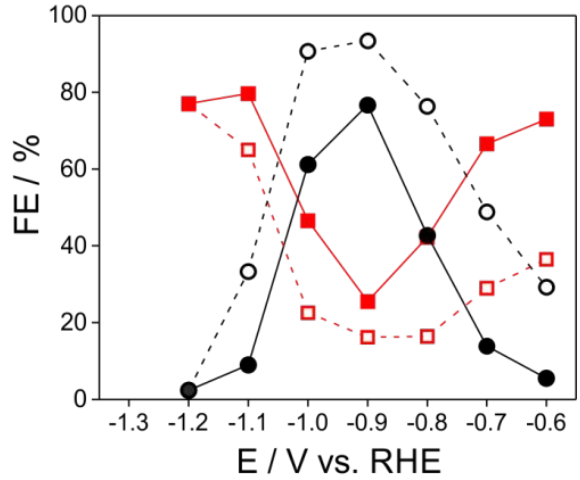

hydrogen (forward run)

hydrogen (backward run)

carbon monoxide (forward run)

O carbon monoxide (backward run) 


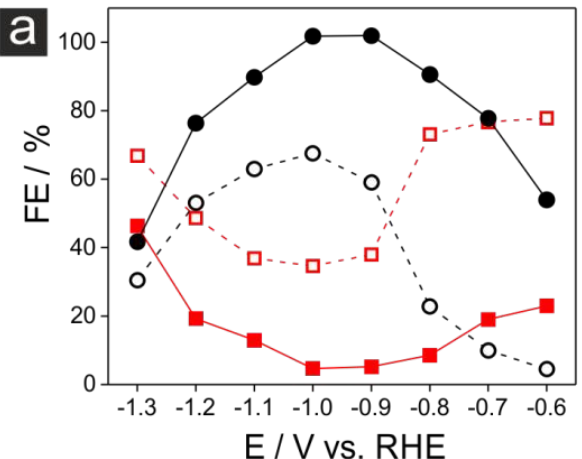

- $\mathrm{CO}$ (activation by ec-1) $\mathrm{H}_{2}$ (activation by ec-l)

O CO (as prepared) $\quad \mathrm{H}_{2}$ (as prepared)

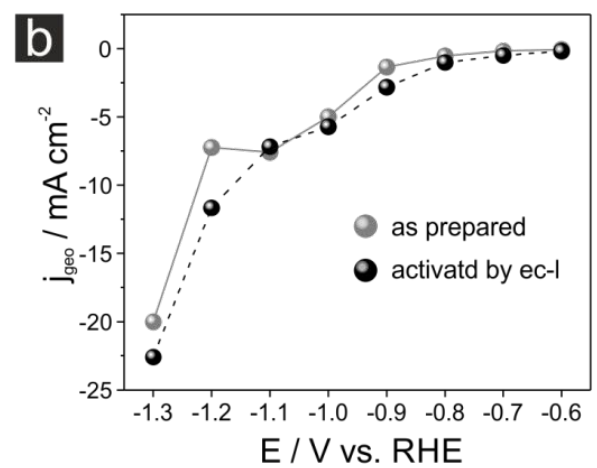

Figure 7. a) $e c-\mathrm{CO}_{2} \mathrm{RR}$ product distribution of $1 \mathrm{~h}$ lasting electrolysis experiments comparing the as prepared Ag-NW catalysts and those pre-treated by an electrochemical looping $\left(E_{\text {vertex }}=-1.3 \mathrm{~V}\right.$ vs RHE, see Figure 3e); b) Steady-state total current densities of the electrolysis experiments which correspond to the data in panel a. 

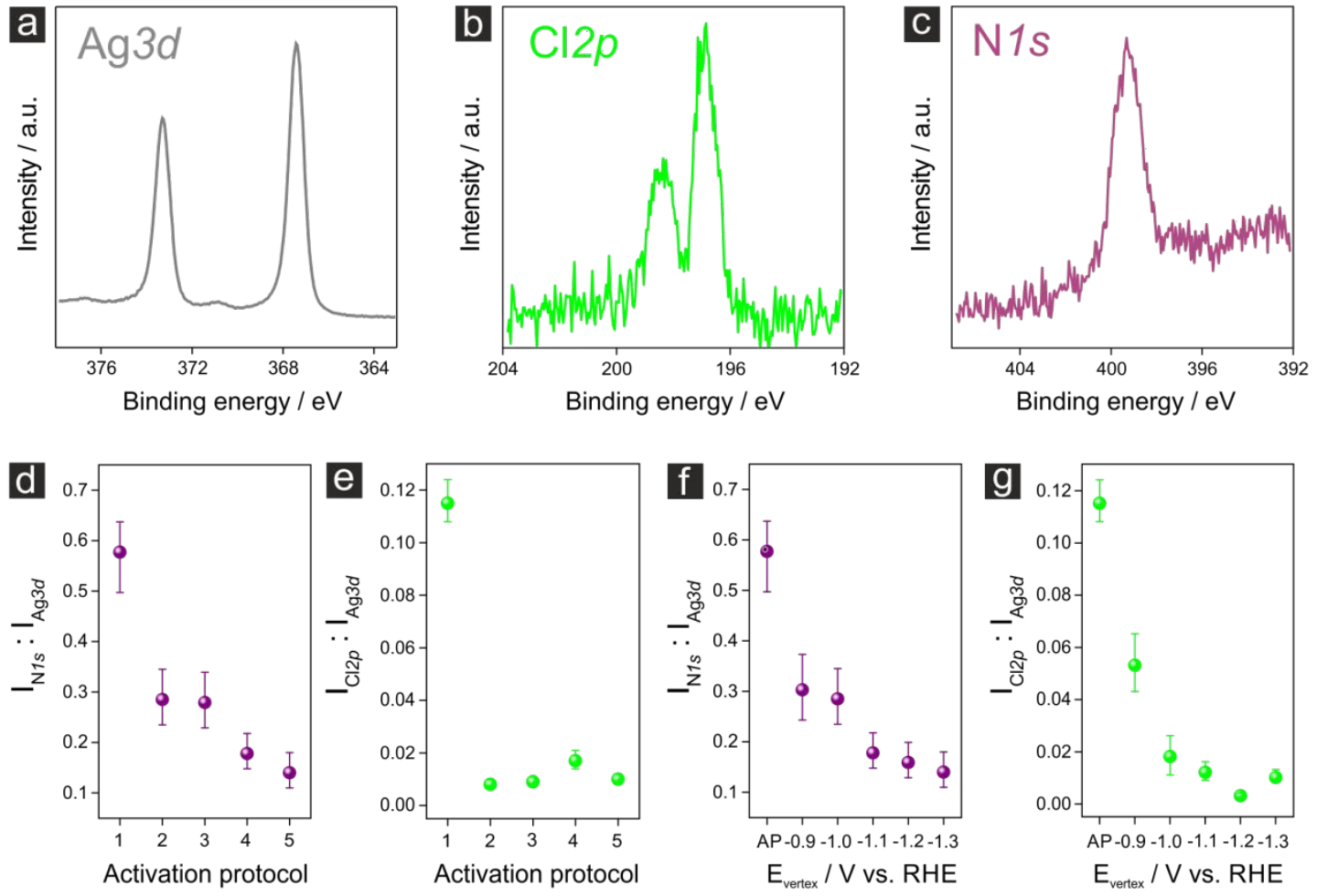

Figure 8. a) - c) Representative XPS spectra of the $\mathrm{Ag} 3 d, \mathrm{Cl} 2 p$ and $\mathrm{N} 1 s$ emissions derived from the Ag-NW catalyst on the GC support electrode (see Figure 2a-c); d) - e) Integrated intensities of the $\mathrm{N} 1 s$ and $\mathrm{Cl} 2 p$ emissions normalized to the corresponding integrated intensity of the $\mathrm{Ag} 3 d$ emission, the digits on the $\mathrm{x}$-axis indicate the respective catalyst activation protocols (1: as prepared; 2: galvanostatic electrolysis in Ar-saturated $\left(\mathrm{CO}_{2}\right.$-free) $0.5 \mathrm{M}$ $\mathrm{KHCO}_{3}$ solution at $\mathrm{j}=-3 \mathrm{~mA} \mathrm{~cm}-2$, the total transferred charge was $\mathrm{Q}=115.3 \mathrm{C} ; \mathbf{3}$ : electrochemical looping (ec-1) in Ar-saturated $0.5 \mathrm{M} \mathrm{KHCO}_{3}$ solution, the vertex potential was $\mathrm{E}_{\text {vertex }}=-1.3 \mathrm{~V}$ vs RHE, the total transferred charge was $\mathrm{Q}=895.9 \mathrm{C}$; 4: galvanostatic electrolysis in $\mathrm{CO}_{2}$-saturated $0.5 \mathrm{M} \mathrm{KHCO}_{3}$ solution at $\mathrm{j}=-3 \mathrm{~mA} \mathrm{~cm}^{-2}$, the total transferred charge was $\mathrm{Q}=115.3 \mathrm{C} ; 5$ electrochemical looping (ec-1) in $\mathrm{CO}_{2}$-saturated $0.5 \mathrm{M} \mathrm{KHCO}_{3}$ solution, the vertex potential was $E_{\text {vertex }}=-1.3 \mathrm{~V}$ vs RHE, the total transferred charge was $\mathrm{Q}=115.3 \mathrm{C}$ (the activation conditions correspond to those in Figure $2 \mathrm{~b}$ ); $\mathrm{f}$ ) $-\mathrm{g}$ ) Integrated intensities of the $\mathrm{N} 1 s$ and $\mathrm{Cl} 2 p$ emissions normalized to the corresponding integrated intensity of the $\mathrm{Ag} 3 d$ emission measured after the electrochemical looping (ec-l) treatment, the respective vertex potentials are indicated on the $\mathrm{x}$-axis (the activation conditions correspond to those in Figure 3 ). 


\section{HER}

$$
2 \mathrm{H}_{2} \mathrm{O}+2 \mathrm{e}^{-} \quad \mathrm{H}_{2}+2 \mathrm{OH}^{-} \quad \mathrm{CO}_{2}+\mathrm{H}_{2} \mathrm{O}+2 \mathrm{e}^{-}
$$

$\mathrm{CO}_{2} \mathrm{RR}$

10

Figure 9. Schematics demonstrating the PVP and $\mathrm{Cl}$ terminated $\mathrm{Ag}$ surface which is still active for the HER (left panel) and the Ag surface which is activated upon CO production through PVP and Cl removal (right panel). 


\section{Graphical abstract}
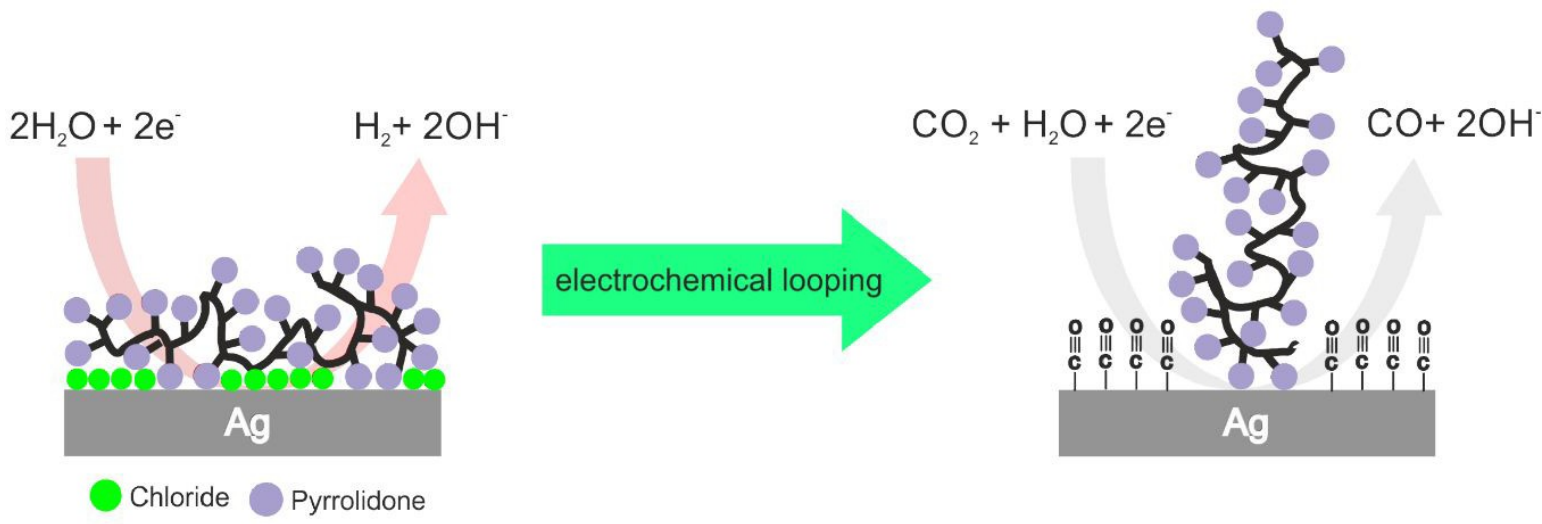